production and increased OC, two arthritis hallmarks. Here, we investigated whether miR-146a was differentially regulated in both Ly6C high and Ly6C $\mathrm{C}^{\text {low }}$ monocyte subsets in healthy and arthritic conditions. Moreover, we developed techniques to deliver siRNA to LyC $6^{\text {high }}$ monocytes in vivo and thus determined the specific impact of miR-146a overexpression in this particular monocyte subset on inflammation and osteoclastogenesis, in the context of arthritis.

Materials and Methods Subset monocytes were isolated from peripheral blood of arthritic and healthy mice by FACS sorting following membrane stainings. Transcriptomic analyses for miRNA expression were performed $(n=6)$ and differential miRNA expression levels were validated on individual samples using multiplex RT-qPCR $(n=13)$. Collagen-induced arthritic (CIA) mice were injected intravenously at disease onset with lipoplex containing either control or miR-146a mimic $(0.5 \mathrm{mg} / \mathrm{kg})$. Arthritis severity was monitored and $\mathrm{OC}$ differentiation was performed by stimulating bone marrow-derived monocytes (BMDM) with M-CSF and RANK$\mathrm{L}$ miR-146a expression levels measured during OC differentiation. TRAP activity, OC numbers and nuclei per OC were quantified.

Results Transcriptomic analyses showed higher expression of miR-146a in Ly6C ${ }^{\text {low }}$ monocytes when compared to Ly6C high monocytes, in both healthy and arthritis mice. In arthritis mice, expression of miR-146a in Ly6C $\mathrm{C}^{\text {high }}$ monocytes is down-regulated as compared to healthy controls. During differentiation of BMDM into OC, miR-146a expression levels are down-regulated on day 2. Enforced expression of miR-146a in Ly6 $\mathrm{C}^{\text {high }}$ monocytes upon intravenous injection of miR-146a-containing lipoplex leads to decreased bone erosion in mouse CIA. This effect was associated with a decreased number of mature OC and TRAP activity, as well as a reduced number of nuclei per OC.

Conclusions Overall, our results show that specific over-expression of miR-146a in Ly6C high monocytes alters OC differentiation and decreased bone erosion in mouse CIA. These data also suggest that Ly6C $\mathrm{C}^{\text {high }}$ monocytes might be the monocyte subset precursor of $\mathrm{OC}$, and that targeting this specific monocyte subset might represent a therapeutic option in the context of arthritis to inhibit bone loss.

\section{A9.18 POTENTIAL THERAPEUTIC APPLICATION OF HUMAN UMBILICAL CORD WHARTON JELLY DERIVED MESENCHYMAL STEM CELLS IN PRIMARY SJÖGREN'S SYNDROME}

doi:10.1136/annrheumdis-2013-203223.18

'Alessia Alunno, 2 Pia Montanucci, 'Sara Caterbi, 'Onelia Bistoni, ${ }^{2}$ Giuseppe Basta, 'Elena Bartoloni, ${ }^{2}$ Teresa Pescara, ${ }^{2}$ llaria Pennoni, 'Riccardo Calafiore, 'Roberto Gerli. ${ }^{1}$ Rheumatology Unit, Department of Clinical and Experimental Medicine; '2Section of Internal Medicine, Endocrine and Metabolic Sciences, University of Perugia, Perugia, Italy

Background and Objectives hUCMS are adult stem cells easy to retrieve in bulk, under acceptable ethical conditions. Their immunemodulatory and pro-differentiation properties have been widely demonstrated. Modulation of the immune system is mediated by both cell contact and soluble factors such as interferon (IFN)- $\gamma$ produced by hUCMS. Very few evidence is currently available with respect to potential therapeutic application of hUCMS in systemic autoimmune disorders. In particular, no data have been published in primary Sjögren's syndrome (pSS) to date. Furthermore, we have recently developed an endotoxin-free alginate matrix which can be used to microencapsulate $(\mathrm{CpS})$ different cell types and transfer them into a non-immunosuppressed host. These microcapsules containing pancreatic islets, upon approval by the Italian Ministry of Health for in vivo use, have been grafted into a cohort of patients with type 1 diabetes mellitus with no adverse effects, while proving to be immunoprotective. On this background, we aimed to assess the in vitro immune system modulation by IFN- $\gamma$ pretreated CpShUCMS on T cells from pSS, with special regard to Th17- and Tregcell subsets.
Materials and Methods Ten pSS patients and 5 healthy donors (HD) were enrolled. Peripheral blood mononuclear cells (PBMC) were obtained by density gradient from heparinised venous blood. Co-cultures of CpS-hUCMS and PBMCs were arranged at different ratios. Lymphocyte proliferation was assessed by CFSE dilution assay. Phenotypic analysis by flow cytometry for regulatory and effector $\mathrm{T}$ cell subpopulations was performed after culture. Real time PCR analysis and the evaluation of culture supernatants for cytokine expression are currently ongoing.

Results CpS-hUCMS were able to inhibit HD and pSS PBMC cell proliferation and this effect was inversely correlated to hUCMS number. Phenotypic analysis revealed a predominant Th17-cell response in SS which was promptly hampered by CpS-hUCMS Moreover, Th1-cell proliferation was fair and reduced Cps-hUCMS FoxP3 and IL-17 expression among CD4 ${ }^{+} \mathrm{T}$ cells was also modulated by CpS-hUCMS, thereby suggesting that a Treg/Th17 rebalance.

Conclusions This is the first study evaluating the effects of hUCMSS on T cells in pSS. It appeared that CpS-hUCMS rebalance Treg/Th17 ratio and therefore may exert therapeutic effects in such disease. Furthermore, it is the first study that employs a new technology of drug delivery which may be applied in vivo in pSS patients.

\section{Others}

\section{A10.1 ANALYSIS OF THE MIGRATORY POTENTIAL OF DERMAL FIBROBLASTS OF PATIENTS WITH SYSTEMIC SCLEROSIS}

doi:10.1136/annrheumdis-2013-203224.1

'S Lefèvre, 'FMP Meier, ${ }^{2} \mathrm{~A}$ Günther, ${ }^{1} \mathrm{U}$ Müller-Ladner, 'E Neumann. 'Dept Internal Medicine and Rheumatology, Justus-Liebig-University Gießen, Kerckhoff Clinic, Bad Nauheim, Germany; ${ }^{2}$ Dept Internal Medicine, Medical Clinic II, Justus-Liebig-University Gießen, Gießen

Background Systemic sclerosis (SSc) is characterised by fibroblastmediated progressive skin fibrosis. Subsequent involvement of internal organs leads to severe impairments of their function and lung fibrosis represents the most common cause of death in SSc. Molecular mechanisms of the spreading of SSc are largely unknown. Recent results showed the migratory potential of rheumatoid arthritis (RA) synovial fibroblasts (SFs). Based on these results, the aim of this study was the analysis of migratory and adhesive properties of SSc dermal fibroblasts (DFs) and their role in SSc-spreading with focus on organ involvement.

Methods SScDFs (in part GFP-transfected) were injected intracutaneously into SCID mice. After 14-25 days, parts of the skin, internal organs and blood were analysed. Besides fluorescence analysis, immunohisto- and -cytochemistry was performed using speciesspecific antibodies to detect human cells. To avoid cross-reactivity of the antibodies, additional real time PCR analysis with primers for human $\beta 2$-microglobulin was performed after RNA isolation out of the respective organs and subsequent reverse transcription.

To analyse and to compare the adhesive behaviour of SScDFs with fibroblasts from other diseases and origins, multi-well culture plates were coated with Matrigel ${ }^{\circledR}(\mathrm{MG})$, growth factor-reduced (GFR) MG, or remained untreated. Cellular adhesion of SScDFs $(n=5)$, RASFs $(n=5)$, RADFs $(n=5)$, SFs $(n=2)$ and DFs of healthy individuals $(n=4)$ was analysed.

Results SScDFs or human cDNA, respectively, were not detected in any internal organ by fluorescence analysis, immunohistochemistry or real time PCR. Human SScDFs and human cDNA were only detectable in the murine skin at the injection site.

Fibroblasts of SSc patients, healthy SFs and DFs showed an increased adhesion to GFR MG compared to MG (SSc: GFR MG: 8.5 fold, MG: 8.2 fold; healthy SFs: GFR MG: 6.6 fold, MG: 4 fold; healthy DFs: GFR MG: 10 fold, MG: 7.6 fold). In contrast a reduction of cellular adhesion of RASFs (GFR MG: 4 fold, MG: 5.4 fold) 
and RADFs (GFR MG: 4.4 fold, MG: 4.8 fold) to GFR MG was observed compared to MG.

Discussion According to present knowledge and in comparison to results obtained from RASFs, SScDFs do not show the ability to migrate from their application site to internal organs. Adhesive properties do not differ from healthy controls. Nevertheless, SScDFs are mediators of organ fibrosis, but it seems that there is no contribution of SScDFs to the spreading of the disease.

\section{A10.2 ANTI-AT1R AND ANTI-ETAR AUTOANTIBODIES IN PATHOGENESIS OF SYSTEMIS SCLEROSIS}

doi:10.1136/annrheumdis-2013-203224.2

${ }^{1}$ Angela Kill, ${ }^{2}$ Mike 0 Becker, ' Jeannine Guenther, ${ }^{3}$ Harald Heidecke, ${ }^{2}$ Duska Dragun, 'Gabriela Riemekasten. 'Charité University Hospital, German Rheumatology Research Center, a Leibniz Institute, Berlin, Germany; ${ }^{2}$ Charité University Hospital, Berlin, Germany; ${ }^{3}$ CellTrend GmbH, Luckenwalde, Germany

Background and Objectives SSc is a prototypic multiorgan disease characterised by vascular damage, autoimmunity and fibrosis with still unkown aetiology. Recently identified functional autoantibodies simultaneously targeting the angiotensin-II type- 1 receptor (AT1R-Abs) and the endothelin-1 receptor type A (ETAR-Abs) were linked to vascular and fibrotic complications in SSc. Presence of both autoantibodies moreover predicted mortality due to cardiopulmonary complications implicating their contribution in SSc pathogenesis. Here, autoantibody mediated effects on endothelial cell activation and their blockade by receptor inhibitors were studied.

Materials and Methods Human microdermal endothelial cells-1 (HMEC-1) and human dermal fibroblasts were treated with IgG from SSc patients containing anti-AT1R and anti-ETAR Abs (SScIgG) or with IgG from healthy donors (NC-IgG) as negative control. In parallel, SSc-IgG treated cells were incubated with AT1R-and ETAR- antagonists alone and in combination. Activation of endothelial cells was assessed by qRT-PCR and sandwich ELISA and of fibroblasts with immunocytochemistry.

Results Human endothelial cells showed increased expression and production of the pro-inflammatory chemokine interleukin-8 (IL-8) upon treatment with SSc-IgG positive for anti-AT1R and antiETAR Abs compared to control treatment with NC-IgG as analysed on mRNA and on protein levels. Furthermore, endothelial cells showed increased expression of the adhesion molecules vascular cell adhesion molecule-1 (VCAM-1) and intercellular adhesion molecule-1 (ICAM-1) with SSc-IgG versus NC-IgG treatment on mRNA levels. Expression of IL-8 and VCAM-1 was significantly decreased by receptor inhibition. Human dermal fibroblasts showed increased collagen 1 production upon treatment with SSc-IgG versus NC-IgG that was also reduced by receptor inhibition.

Conclusions Our data sugests a direct involvement of anti-AT1R and anti-ETAR Abs in endothelial cell and fibroblasts activation that is mediated by AT1R and ETAR activation. Increased expression of IL-8 and of VCAM-1 and ICAM-1 indicate a direct endothelial cell activation and inflammation. Increased collagen 1 production indicates fibroblast activation and pro-fibrotic events. Therefore, anti-AT1R and anti-ETAR Abs induce pro-inflammatory and profibrotic events and could be directly involved in the pathogenesis of SSc. In vivo experiments are underway to analyse anti-AT1R and anti-ETAR Abs mediated pathogenic events in SSc.

\section{A10.3 ARTHRITIS IN PATIENTS WITH IDIOPATHIC INFLAMMATORY MYOPATHIES}

doi:10.1136/annrheumdis-2013-203224.3

\footnotetext{
1,2Martin Klein, 1,2Heřman Mann, 'Petra Hánová, 1,2Lenka Pleštilová, 1,2Jakub Závada, 1,2 Jiři Vencovský. 'Institute of Rheumatology, Prague, Czech Republic; '2Department of rheumatology $1{ }^{\text {st }}$ Faculty of Medicine, Charles University, Prague, Czech Republic
}

Background and Objectives Arthritis in idiopathic inflammatory myopathies (IIM) is frequently observed in clinical practise, but, to our knowledge, there is lack of systemic reports of prevalence and/ or characteristics of arthritis in myositis patients. The aim of this study is to determine prevalence of arthritis in IIM patients; it's relation to the course of the muscle disease; characteristics of arthritis with respect to seriousness, distribution and extent as well as it's relation to autoantibody profiles and others disease's characteristics.

Materials and Methods In this cross-sectional study, clinical aspects of disease, history of arthritis and autoantibody profiles were obtained from 106 consecutive patients with definite diagnosis of IIM. In all of them the 68-joint index was investigated. In 55 IIM patients and in 60 control patients with rheumatoid arthritis (RA) German Ultrasound Score 7 (US-7) was performed.

Results Arthritis at any time of course of myositis occurred in 65 patients (61.3\%); 42 had arthritis at the beginning of myositis (in 22 patients before and in 16 together with onset of muscle weakness). 52 patients presented arthritis at clinical examination (25 poly-, 17 oligo-, and 10 with monoarthritis). Most frequently affected joints were wrists $(21.7 \%)$ and shoulders, metacarpophalangeal, and proximal interphalangeal joints (20.8\%). From 29 anti-Jo-1 positive patients 28 had arthritis and significant association between arthritis and anti-Jo-1 positivity was found ( $p<0.0001) .39$ out of 55 $(70.9 \%)$ patients had Gray-Scale (GS) synovitis on ultrasound; in 34 of them also Power-Doppler (PD) positivity was found. Only 4 patients had ultrasonographic tenosynovitis and 3 had bony erosions. From 60 RA patients 57 (95\%) had GS synovitis, which was $\mathrm{PD}$-active in 54 . Tenosynovitis was found in 25 patients. $25 \mathrm{RA}$ patients had one or more joint erosions. Mean US-7 score as well as scores of individual joints or modality subscores were significantly lower in IIM than in RA, but, when compared only those patients with positive findings, the differences were found in total US-7 score, and GS-synovitis and PD-tenosynovitis subscores, but not in PD-synovitis, GS-tenosynovitis as well as in scores of individual joints.

Conclusions Our data suggest that arthritis is common feature of myositis. It is often present at the beginning of muscular manifestation of disease, or it even precedes the onset of muscle weakness. Most common presentation is symmetrical, non erosive polyarthritis affecting particularly wrists, shoulders, metacarpophalangeal and proximal interphalangeal joints of the hands. We confirmed strong association of arthritis with anti-Jo-1 antibody. Ultrasound investigation of joint involvement in IIM shows less frequent involvement than in RA, but comparable activity of synovitis measured by PD in affected joints. IIMs have less erosions and tenosynovitis than RA.

\section{A10.4 AUTOMATED AND STANDARDISED ANALYSIS FOR HIGH DIMENSIONAL CYTOMETRIC DATA PROVIDES NEW OPTIONS FOR COMPLEX CELL-ASSOCIATED BIOMARKER SCREENING}

doi:10.1136/annrheumdis-2013-203224.4

'Till Sörensen, '2Ursula Schulte-Wrede, 'Silvia Pade, ${ }^{2}$ Heike Hirseland, 'Gerd Burmester, ${ }^{2}$ Andreas Radbruch, ${ }^{2}$ Andreas Grützkau, 'Thomas Häupl. 'Dept. of Rheumatology and Clinical Immunology, Charité University Medicine, Berlin; ${ }^{2}$ German Arthritis Research Center, Berlin

Background and Objectives Flow cytometry (FCM) is widely used in clinical research and offers rapid and quantitative characterisation at single cell level. Traditional analysis is a semiautomated, time-consuming process of gating and successive 2-D projections, influenced by investigator-specific settings. With an increasing number of parameters for multiplexing, the manual analysis step is most limiting and impedes high throughput analysis in FCM. 